\title{
In Vitro Activation of Valproate Glucuronidation by Carbapenem Antibiotics
}

\author{
Hitomi Mori and Takaharu Mizutani* \\ Department of Drug Metabolism and Disposition, Graduate School of Pharmaceutical Sciences, Nagoya City University, Nagoya \\ 467-8603, Japan
}

(Received January 12, 2007; Accepted February 1, 2007; Published online February 5, 2007)

\begin{abstract}
The serum concentration of valproic acid (VPA) in epilepsy patients decreased by the administration of carbapenem antibiotics, such as meropenem, panipenem or imipenem, to a sub-therapeutic level. Studies to explain the decrease were carried out using almost rats by the following steps: absorption of VPA in the intestine, glucuronidation in the liver, disposition in blood and renal excretion. It is difficult to consider the inhibition of intestinal absorption, because carbapenem antibiotics are intravenously administered and do not reach the intestine at an effective concentration. The liver is the key organ for the decrease of VPA concentration by carbapenem antibiotics, because it has been reported that no decrease of the VPA level by carbapenem was found in hepatectomized rats. The most likely mechanism in liver is the activation of UDP-glucuronosyltransferase by carbapenem antibiotics. We found a 35\% increase of VPA-glucuronidation activity by the pre-incubation of human liver microsomes with meropenem. We estimated that this increase fully compensates for the decrease of serum VPA level by carbapenem antibiotics in patients. Meanwhile, we could not find the in vitro activation of CYP2A6, CYP3A4, P-glycoprotein (P-gp, MDR1) and Multidrug resistance-associated protein 2 by pre-incubation with carbapenem antibiotics. Thus, we considered that the activation of VPA glucuronidation by carbapenem antibiotics is a key point in the decrease of plasma VPA level.
\end{abstract}

Key words — carbapenem antibiotics, meropenem, valproic acid, drug interaction, CYP3A4, UDP -glucuronosyltransferase, P-glycoprotein, Multidrug resistance-associated protein 2

\section{INTRODUCTION}

Most xenobiotics, such as drugs, non-nutrient chemicals in foods, and pollutants are metabolized in the human body. ${ }^{1)}$ Phase I drug-metabolizing enzymes functionalize lipophilic drugs by adding active residues such as $\mathrm{OH}$ to the drugs. Phase II metabolizing enzymes conjugate with watersoluble substances, such as uridine $5^{\prime}$-diphosphate (UDP)-glucuronic acid (UDPGA) for UDPglucuronosyltransferase (UGT), ${ }^{2,3)}$ PAPS for sulfotransferase, ${ }^{4)}$ and glutathione for glutathione S-transferase, to further functionalize the drugs. These soluble conjugates are excreted in the bile and the urine by transporters. ${ }^{5)}$ Drug-drug interactions are reported in patients administered several

\footnotetext{
*To whom correspondence should be addressed: Department of Drug Metabolism and Disposition, Graduate School of Pharmaceutical Sciences, Nagoya City University, Nagoya 467-8603 Japan. Tel.\&Fax: +81-52-836-3490; E-mail: mizutani@phar.nagoya-cu.ac.jp
}

drugs. A typical example is the transcriptional induction of CYP3A4 by rifampicin. Ethinylestradiol used for contraception was rapidly metabolized by a high amount of CYP3A4 induced by rifampicin, and unexpected pregnancy occured. ${ }^{6)}$ In addition to transcriptional gene activation of drug-metabolizing enzymes, molecular mechanisms of activation by stabilization, such as mRNA stabilization (CYP1A2 by 3-methylcholanthrene) and protein stabilization (CYP2E1 by ethanol, in part) and/or the increase of enzymes and/or cofactors supporting drug-metabolizing enzymes were found. However, there are drug-drug interactions, which are difficult to explain by the mechanisms described above. One is that the serum valproic acid (VPA) level in epilepsy patients decreases by the administration of carbapenem antibiotics (CPs), such as meropenem (MEPM), panipenem (PAPM), imipenem (IPM) and biapenem (BIPM). This decrease does not depend upon the transcriptional gene activation of some drug-metabolizing enzymes. 
A decade ago, the plasma level of VPA (1-4 g dose) in an epilepsy patient rapidly decreased by the administration of panipenem, a carbapenem antibiotic, which was used to treat pneumonia and this decrease resulted in clinical failure. ${ }^{7)}$ VPA is excreted from the kidney after conjugation with glucuronide by UGT in the liver. In blood, VPA is the bound state with plasma protein and carbapenem antibiotics are not bound with plasma protein. The structure of VPA, capbapenem antibiotics, and related other drugs used in therapy of epileptic patients or this study are shown in Fig. 1.

The main purpose of this study is to clarify the mechanism of the decrease of VPA level in blood by carbapenem antibiotics. Studies to explain the decrease were carried out using almost rats by the following steps (Table 1): ${ }^{8-15}$ absorption of VPA in the intestine, glucuronidation in the liver, disposition in blood and renal excretion. In the intestine of absorption step, there are two points: inhibition of the intestinal transporter for VPA absorption by carbapenem antibiotics, and the decrease of $\beta$ glucuronidase supplied from enteric bacteria, which were killed by antibiotics. This means that the decrease of VPA originated from VPA-glucuronide (Glu), relating to entero-hepatic circulation. The second key step is in the liver, because no decrease of the VPA level by carbapenem antibiotics was found in hepatectomized rats. Two points in the liver explain this decrease: first, decrease of the UDPGA level by carbapenem antibiotics. UDPGA is a cofactor for glucuronidation of VPA by UGT. It is difficult to consider the induction of UGT1A6, because the decrease of VPA was found in $15 \mathrm{~min}$ after administration of carbapenem antibiotics to rat. ${ }^{12)}$ Second, the inhibition of $\beta$-glucuronidase in liver slices by carbapenem antibiotics and the decreased VPA amount liberated from VPA-Glu. The third step is the blood distribution of VPA. A small amount of plasma VPA transferred to erythrocytes by the inhibition of transporters (Mrp4), which efflux VPA from erythrocytes, by carbapenem antibi-<smiles>CCCC(CCC)C(N)=O</smiles>

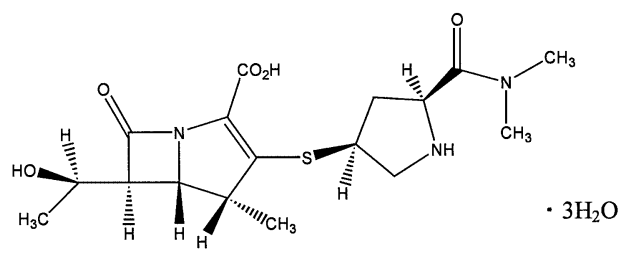

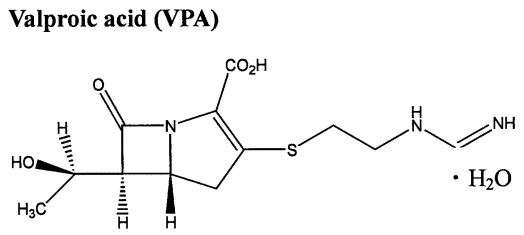

Imipenem (IPM)

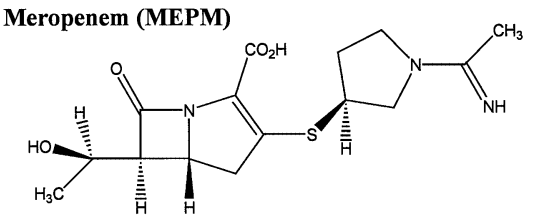

Panipenem (PAPM)

Fig. 1. Chemical Structure of Valproic Acid, Meropenem, and Other Drugs

Table 1. Summary of the Possible Mechanisms of VPA Decrease by Carbapenem Antibiotics

\begin{tabular}{lllr}
\hline \hline Target organ & Mechanism proposed & Drug used & Reference \\
\hline Intestine & Decrease of $\beta$-glucuronidase level by antibiotics & 8 \\
& Inhibition of intestinal absorption and Caco-2 cell monolayer & PAPM & 9 \\
Liver & No-reaction with hepatectomized rats & PAPM & 10 \\
& Elevation of UDPGA concentration & PAPM & 11 \\
& Increase of synthesis and inhibition of hydrolysis of VPA-Glu & PAPM, MEPM & 12 \\
& In vitro activation of UDP-glucuronosyltransferase & MEPM & This work \\
Blood & Accumulation of VPA in erythrocytes & PAPM, IPM & 13 \\
& Inhibition of efflux of VPA from erythrocytes by Mrp4 & PAPM, IPM, MEPM & 14 \\
Kidney & Elevation of excretion of VPA-Glu & MEPM & 15 \\
\hline
\end{tabular}


otics. The increase of renal excretion of VPA as VPA-Glu depends on the increase of VPA-Glu level by UGT and the decrease was not found in nephrectomized rats.

In this study we show the in vitro activation of glucuronidation of VPA by carbapenem antibiotics. This activation was performed by pre-incubation of human liver microsomes with carbapenem antibiotics.

\section{MATERIALS AND METHODS}

Materials — Pooled human liver microsomes (HLM) and P-glycoprotain (P-gp, MDR1) are purchased from Gentest (Woburn, MA, U.S.A.). Human MRP2 membranes were a gift from Genomembrane (Yokohama, Japan). P-gp and MRP2 are products prepared in Sf9 cell membrane using a baculovirus expression system. Bovine microsomes and murine microsomes were prepared by the standard method. ${ }^{16)}$ Sodium valproate (VPA), probenecid, sodium ampicillin, UDPglucuronic acid, p-nitrophenol (p-NP), and verapamil hydrochloride were products of Sigma Chemical Co. (St. Louis, MO, U.S.A.). Sodium orthovanadate and glycoletherdiamine- $N, N, N^{\prime}, N^{\prime}$ tetraacetic acid (EGTA) obtained from Wako Pure Chemicals (Osaka, Japan). MEPM and BIPM are from Sumitomo Pharmaceutical Co. (Osaka, Japan) and Meiji Pharmaceutical Co. Ltd (Tokyo, Japan), respectively. IPM and streptomycin sulfate are from Banyu Pharmaceutical Co (Tokyo, Japan). PAPM and nitrilotriacetic acid are from Sankyo Co. Ltd. (Tokyo, Japan) and Tokyo Kasei Co (Tokyo, Japan), respectively. VPA-Glu is a gift from Sankyo Co. Ltd (Tokyo, Japan). $\left[\gamma^{-32} \mathrm{P}\right] \mathrm{ATP}$ and $\left[{ }^{14} \mathrm{C}\right] \mathrm{UDPGA}$ are from Institute of Isotopes Co. (Budapest, Hungary) and American Radiolabeled Chemicals Inc. (St. Louis, MO, U.S.A.), respectively.

Activity Measurement of VPA Glucuronidation with HLM — VPA glucuronidation by UDPglucuronosyltransferase was determined according to the previous report. ${ }^{16)}$ HLM not activated in TritonX-100 was used except experiments described the addition of Triton X-100. HLM $(1 \mu \mathrm{l})$ was pre-incubated at $0^{\circ} \mathrm{C}$ or $37^{\circ} \mathrm{C}$ for $30 \mathrm{~min}$ in a mixture $(49 \mu \mathrm{l})$ composed of $20 \mathrm{mM}$ Tris-maleate (pH 7.4), $1 \mathrm{mM} \mathrm{MgCl} 2,2 \mathrm{mM}\left[{ }^{14} \mathrm{C}\right] \operatorname{UDPGA}(2 \mu \mathrm{Ci})$ and MEPM at appropriate concentration. The reaction was started by addition of $1 \mu \mathrm{l}$ of $100 \mathrm{mM}$ VPA (final concentration $2 \mathrm{mM}$ ) and incubated for

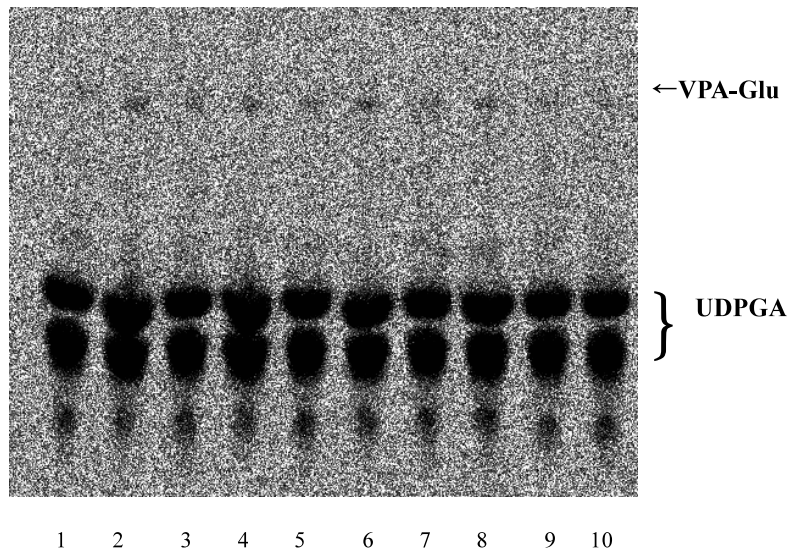

Fig. 2. Autoradiography Showing the Activation of VPA Glucuronidation by MEPM

The conditions of pre-incubation are as follows; odd lanes are at $37^{\circ} \mathrm{C}$ and even lanes at $0^{\circ} \mathrm{C}$. Lanes 1 and 2 are $12 \mu \mathrm{M}$ MEPM and lanes 3 and 4 are $120 \mu \mathrm{M}$ MEPM. Lanes 5 and 6 are $20 \mu \mathrm{M}$ BIPM and lanes 7 and 8 are $200 \mu \mathrm{M}$ BIPM. Lanes 9 and 10 are no carbapenem antibiotics.

$30 \mathrm{~min}$ at $37^{\circ} \mathrm{C}$. The reaction was stopped by the addition of $110 \mu \mathrm{l}$ cold ethanol and the mixture was centrifuged. The supernatant was dried by centrifuged-evaporator and then dissolved in $10 \mu \mathrm{l}$ of $70 \%$ ethanol and the solution was spotted on a TLC plate (Merck Silica Gel 60). The plate was developed in a solvent ( $n$-butanol : $\mathrm{dH}_{2} \mathrm{O}$ : acetone : glacial acetic acid : 30\% ammonium $=70: 60$ : $50: 18: 1.5)$. $\left[{ }^{14} \mathrm{C}\right]$ VPA-Glu was moved to near the top of TLC plate. $\left[{ }^{14} \mathrm{C}\right]$ UDPGA of the cofactor of UGT was at the middle of TLC plate as shown in Fig. 2. The radioactivity $\left[{ }^{14} \mathrm{C}\right]$ UDP-Glu on the plate was measured with a bio-image analyzer Fuji BAS 2500.

The influences of MEPM for glucuronidation of p-NP and androsterone, instead of VPA, were carried out according to the same method of VPA glucuronidation. The glucuronidation products with p-NP and androsterone were also analyzed by the same method described above. The influence of streptomycin and ampicillin instead of MEPM on VPA glucuronidation was also studied as described above.

Measurement of the Activity of Transporters — The activity of P-gp was determined by the measurement of inorganic phosphate liberator from ATP. We used the radioactive $\left[\gamma_{-}{ }^{32} \mathrm{P}\right] \mathrm{ATP}$ for the measurement of inorganic phosphate. This method is different from the method with the standard phospho-morybdate method. ${ }^{17)}$ Our method is based on the fact that $\left[\gamma_{-}{ }^{32} \mathrm{P}\right]$ ATP absorbs on charcoal and inorganic phosphate does not adsorb 
on charcoal. ${ }^{18)}\left[\gamma_{-}{ }^{32} \mathrm{P}\right]$ ATP of a product from a maker was used further purification. We analyzed the product $\left[\gamma_{-}{ }^{32} \mathrm{P}\right]$ ATP by chromatography on a column of AG1 (BioRad). The reaction mixture $(19 \mu \mathrm{l})$ containing P-gp $(1 \mu \mathrm{l}, 5 \mu \mathrm{g})$ or MRP2 $(5 \mu \mathrm{l}$, $100 \mu \mathrm{g})$ in $50 \mathrm{mM}$ Tris-MES, $2 \mathrm{mM}$ EGTA, $1 \mathrm{mM}$ $\left.{ }^{32} \mathrm{P}\right]$ ATP $(0.2 \mu \mathrm{Ci}), 50 \mathrm{mM} \mathrm{KCl}, 2 \mathrm{mM}$ DTT were pre-incubated with MEPM at appropriate concentration for $30 \mathrm{~min}$ at $0^{\circ} \mathrm{C}$. The reaction was started by addition of $1 \mu \mathrm{l}$ of verapamil, VPA or VPA-Glu. The incubation was done for $30 \mathrm{~min}$ at $37^{\circ} \mathrm{C}$. The reaction was stopped by addition of $40 \mu \mathrm{l}$ of $10 \%$ charcoal (Norit A) in $0.1 \mathrm{M}$ phosphate buffer, followed by centrifugation at $8000 \mathrm{rpm}$. Charcoal binding ATP was removed by this centrifugation and the supernatant containing ${ }^{32} \mathrm{P}$ inorganic phosphate was applied to a filter paper and dried. The radioactivity of ${ }^{32} \mathrm{P}$ on the filter paper was analyzed using the radio-image analyzer Fuji BAS 2500.

Measurement of the Activity of Cytochrome $\mathbf{P 4 5 0}$ _ The influence of carbapenem antibiotics on CYP2A6 and CYP3A4 were studied with HLM and bovine liver microsomes. The activity of CYP2A6 was measured by the previous report. ${ }^{19,20)}$ HLM $(4 \mu \mathrm{g})$ was preincubated with MEPM in $15 \mu \mathrm{l}$ at $0^{\circ} \mathrm{C}$ for $30 \mathrm{~min}$. The reaction was started by addition of the reaction mixture $(15 \mu \mathrm{l})$ composed of $3 \mathrm{mM} \mathrm{MgCl} 2,1.3 \mathrm{mM} \mathrm{NADP}{ }^{+}$, $3.3 \mathrm{mM}$ glucose-6-phosphate, $0.4 \mathrm{U}$ glucose-6phosphate dehydrogenase, $10 \mathrm{mM}$ coumarin and $0.1 \mathrm{M}$ Tris- $\mathrm{HCl}(\mathrm{pH}$ 7.4). The mixture was incubated for $30 \mathrm{~min}$ at $37^{\circ} \mathrm{C}$ and the reaction was stopped by addition of $70 \mu \mathrm{l}$ of cold ethanol. The solution was centrifuged and the supernatant was mixed with $700 \mu \mathrm{l}$ of $0.1 \mathrm{M}$ Tris- $\mathrm{HCl}(\mathrm{pH} 9)$ and the mixture $200 \mu \mathrm{l}$ was measured by ARVO 1400 by fluorometry in triplicate. The amount of the product 7-hydroxycoumarin from coumarin was measured with ALVO 1400. The activity of CYP3A4 was measured with an assay kit for CYP3A4, by the similar method with that of CYP2A6, except 7-benzyloxytrifluoromethylcoumarin (67 $\mu \mathrm{M})$ was used instead of coumarin. Fluorometry was done excitation $409 \mathrm{~nm}$ and emission $530 \mathrm{~nm}$ for CYP3A4, and excitation $399 \mathrm{~nm}$ and emission $460 \mathrm{~nm}$ for CYP2A6. 7Hydroxytrifluoromethylcoumarin was used as a standard of product with CYP3A4.

Statistical Analyses — Experiment was done the sample number $n=5-6$ of each point. Statistical significance was calculated by Student's $t$-test and * indicates $p<0.05$. Each point indicates the mean \pm S.D.

\section{RESULTS}

\section{In Vitro Activation of VPA-glucuronidation by Carbapenem Antibiotics}

We investigated the influence of preincubation with MEPM and BIPM. In preliminary experiments, we studied the influence at several concentrations. $12 \mu \mathrm{M}$ or $120 \mu \mathrm{M}$ MEPM and $20 \mu \mathrm{M}$ or $200 \mu \mathrm{M}$ BIPM at $0^{\circ} \mathrm{C}$ or $37^{\circ} \mathrm{C}$. MEPM and BIPM did not activate UGT at high concentration at $37^{\circ} \mathrm{C}$, but at low concentration at $0^{\circ} \mathrm{C}$ (about $40 \%$ increase of UGT activity without drugs) as shown in Fig. 2. The concentration of these bands of VPA-Glu in lanes $1,3,5,7$ at $37^{\circ} \mathrm{C}$ were low but those of lanes 2, 4, 6, 8 at $0^{\circ} \mathrm{C}$ were strong in Fig. 2. The density of lane 2 of $12 \mu \mathrm{M}$ MEPM was higher than that of lane 4 of $120 \mu \mathrm{M}$ MEPM. These concentrations $12 \mu \mathrm{M}$ MEPM and $20 \mu \mathrm{M}$ BIPM are near the in vivo concentrations of those drugs by therapeutic dose.

We then confirmed the above experiments with sample number 6 and the results are shown in Fig. 3. We studied the influence with carbapenem antibiotics, such as MEPM, PAPM, IPN and BIPM. The preincubation was done at $0^{\circ} \mathrm{C}$ for $30 \mathrm{~min}$ according to the results in Fig. 2 and then the reaction started by addition of VPA, like the above

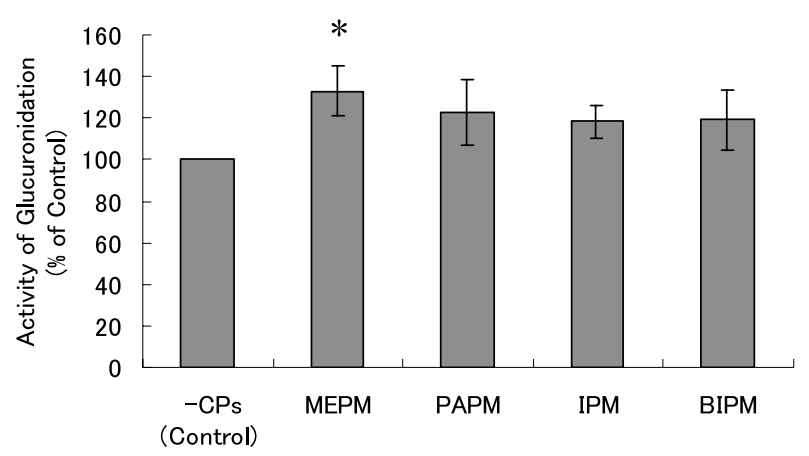

Fig. 3. Activation of in Vitro Valproate Glucuronidation by Carbapenem Antibiotics

Pooled human liver microsomes $(20 \mu \mathrm{g}, 1 \mu \mathrm{l}$ of $20 \mathrm{mg} / \mathrm{ml}$ ) were pre-incubated at $0^{\circ} \mathrm{C}$ for $30 \mathrm{~min}$ in the absence or the presence of $12 \mu \mathrm{M}$ MEPM, $15 \mu \mathrm{M}$ PAPM, $16 \mu \mathrm{M}$ IPM or $20 \mu \mathrm{M}$ BIPM in the pre-incubation mixture containing $2 \mathrm{mM}\left[{ }^{14} \mathrm{C}\right]$ UDPGA, $20 \mathrm{mM}$ Trismaleate at $\mathrm{pH} 7.4$ and $1 \mathrm{mM} \mathrm{MgCl}$. The reaction was started by the addition of VPA at the final concentration $2 \mathrm{mM}$. The mixture was incubated for $30 \mathrm{~min}$ at $37^{\circ} \mathrm{C}$. Then $\left[{ }^{14} \mathrm{C}\right]$ VPA-Glu was analyzed by TLC according to the method described by [16]. There were 6 samples in the experiment. Each points indicate the mean \pm SD and statistical significance was calculated by Student's $t$-test. 
experiment with MEPM. The activities of VPA glucuronidation were increased to $134 \pm 20.4 \%$ at $12 \mu \mathrm{M}$ MEPM, $122.6 \pm 16.0 \%$ at $15 \mu \mathrm{M}$ PAPM, $118.3 \pm 8.1 \%$ at $16 \mu \mathrm{M}$ IPM and $119.2 \pm 14.5 \%$ at $20 \mu \mathrm{M}$ BIPM, compared to the activity as $100 \%$ in the absence of carbapenem antibiotics. The significant increase of the activity with MEPM was confirmed by the repeated experiments (data not shown). The increase (35\%) of the activation by MEPM was slightly stronger than those of PAPM, IPM and BIPM. The $V \max$ value of VPA for UGT1A6 obtained by us is $2.5 \mathrm{nmol} / \mathrm{min} / \mathrm{mg}$ of human liver microsomal protein. The amount of microsomal protein in the liver $(1000 \mathrm{~g})$ is approximately $100 \mathrm{~g}$. The amount of VPA-Glu produced by the liver for $60 \mathrm{~min}$ was $2.5 \mathrm{nmol} \times 60 \mathrm{~min}$ $\times 100000 \mathrm{mg}=15 \mathrm{mmol}$. This $15 \mathrm{mmol} \mathrm{VPA}$ is $2500 \mathrm{mg}$. The $35 \%$ increase of UGT activity corresponds to about $875 \mathrm{mg}(2500 \mathrm{mg} \times 0.35)$ for $1 \mathrm{hr}$. The therapeutic amount of administration of VPA is $400-1200 \mathrm{mg} /$ day and this amount is able to be glucuronized by the $35 \%$ increase of UGT activity. Thus, the $20-35 \%$ activation of VPA conjugation by carbapenem antibiotics in Fig. 3 fully explained the decrease of serum VPA concentration and must influence on total clearance of VPA.

We also confirmed dose-dependency of the in

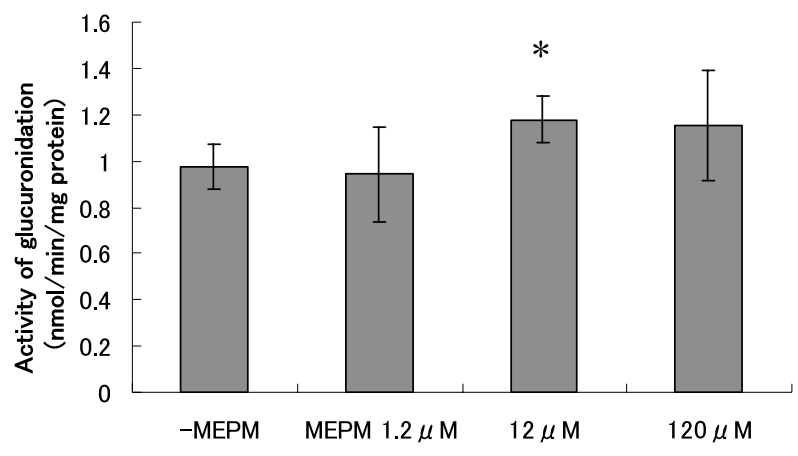

Fig. 4. Dose Dependency of the Activation of VPA Glucuronidation by MEPM

Experimental conditions are the same in Fig. 3. The MEPM concentrations used were $1.2,12$, and $120 \mu \mathrm{M}$. vitro activation by MEPM as shown in Fig. 4. MEPM activated at $12 \mu \mathrm{M}$ and $120 \mu \mathrm{M}$ and not activated at $1.2 \mu \mathrm{M}$. The concentration $12 \mu \mathrm{M}$ was near the therapeutic level estimated from therapeutic dose in patients. Thus, it is possible that carbapenem antibiotics activate the activity of UGT1A6 in human liver to produce VPA-Glu, which is excreted from urine.

We studied the activation of other ligands, such as p-NP and androsterone by MEPM. Table 2 shows that glucuronidation of p-NP by UGT1A6 and androsterone by UGT2B7 are increased by activation by preincubation in $12 \mu \mathrm{M}$ MEPM to $132.7 \%$ and $116.1 \%$, respectively. Thus, MEPM activates UGT2B7 as well as UGT1A6. It is possible that this activation is dependent on the phenomenon like the activation of Triton X-100. Therefore, we examined the influence on VPA glucuronidation by Triton X100. The results were shown in Table 2. At $0.025 \%$ Triton X-100, UGT was activated and the activity was the same level in the presence or absence of MEPM, $2.349 \pm 0.098$ or $2.328 \pm 0.016 \mathrm{nmol} / \mathrm{min} / \mathrm{mg}$ protein, respectively. Activation level by Triton X100 was approximately 2 -fold and the activation level by MEPM was about 1.3-fold. We could not find the additional effect by MEPM to the activation by Triton X-100. Thus, we thought that the mechanism of activation by MEPM differed from detergent property by Triton X-100.

Next, we studied the influence of other antibiotics, such as streptomycin and ampicillin, in VPA glucuronidation. In the presence of at $28 \mu \mathrm{M}$ streptomycin, the activity of VPA glucuronidation was $1.182 \pm 0.047 \mathrm{nmol} / \mathrm{min} / \mathrm{mg}$ protein, compared to the value of $1.329 \pm 0.086 \mathrm{nmol} / \mathrm{min} / \mathrm{mg}$ protein in the absence of streptomycin. Meanwhile, in the presence of $27 \mu \mathrm{M}$ ampicillin, the activity was $1.148 \pm 0.073 \mathrm{nmol} / \mathrm{min} / \mathrm{mg}$ protein, different from the value $0.986 \pm 0.068 \mathrm{nmol} / \mathrm{min} / \mathrm{mg}$ protein in the absence of ampicillin. This difference (16\%) is small but significant between them.

Table 2. The Influence of MEPM on the Conjugation

\begin{tabular}{lccc}
\hline \hline Substrate & \multicolumn{3}{c}{ Activity $(\mathrm{nmol} / \mathrm{min} / \mathrm{mg}$ protein, $n=6)$} \\
\cline { 2 - 4 } & - MEPM & $+12 \mu \mathrm{M} \mathrm{MEPM}$ & $\%$ ratio $(+/-)$ \\
\hline$p$-Nitrophenol & $5.286 \pm 0.516$ & $7.015 \pm 0.544$ & 133 \\
Androsterone & $1.070 \pm 0.045$ & $1.242 \pm 0.120$ & 116 \\
VPA (-Triton X-100) & $1.069 \pm 0.196$ & $1.271 \pm 0.046$ & 119 \\
VPA (in 0.025\% Triton X-100) & $2.328 \pm 0.016$ & $2.349 \pm 0.098$ & 101 \\
\hline
\end{tabular}


Table 3. VPA and VPA-Glu are Not Substrates of P-gp

\begin{tabular}{lc}
\hline \hline Conditions (Concentration) & Activity (nmol/min/mg protein of P-gp) \\
\hline Control (blank, -substrate $)^{a)}$ & $33.78 \pm 0.237$ \\
VPA $(33 \mu \mathrm{M})$ & $31.97 \pm 1.019$ \\
VPA $(1.67 \mathrm{mM})$ & $30.59 \pm 2.110$ \\
VPA-Glu $(2 \mu \mathrm{M})$ & $34.23 \pm 0.685$ \\
VPA-Glu $(20 \mu \mathrm{M})$ & $33.96 \pm 0.706$ \\
VPA-Glu $(0.2 \mathrm{mM})$ & $33.03 \pm 1.106$ \\
VPA-Glu $(2 \mathrm{mM})$ & $31.26 \pm 0.828$ \\
VPA-Glu $(0.2 \mathrm{~m})+$ MEPM $(12 \mu \mathrm{M})$ & $33.52 \pm 1.200$ \\
Verapamil $(1 \mathrm{mM})$ & $54.57 \pm 1.202$ \\
\hline
\end{tabular}

a) $\left[{ }^{32}\right.$ P]ATP contained some impurities of inorganic phosphate.

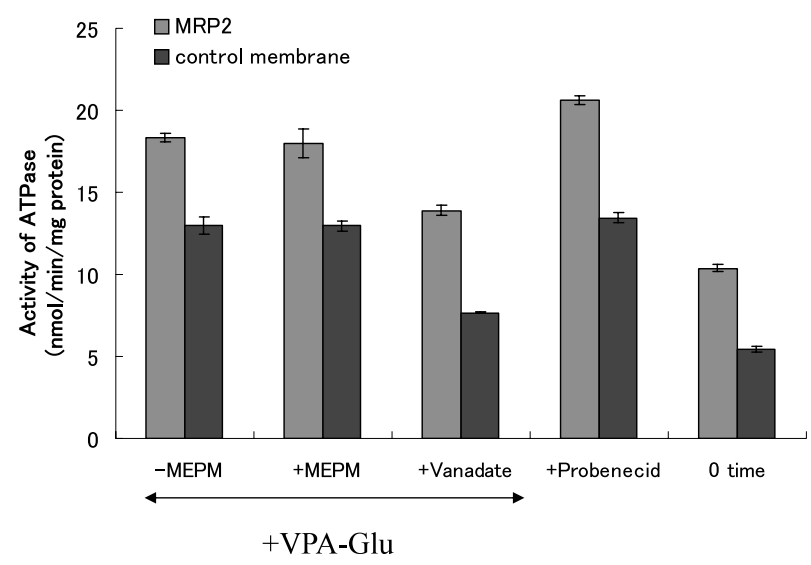

Fig. 5. No Activation of MRP2 by MEPM

MRP2 activity was shown by ATPase activity and compared the results obtained with control membranes not expressing MRP2. Those conditions were described in Methods. Those concentrations of MEPM and VPA-Glu are 12 and $200 \mu \mathrm{M}$, respectively.

\section{No Activation of P-gp and MRP2 by MEPM}

We studied the influence of MEPM on the P-gp activity. The main purpose of this study is to clarify the mechanism for carbapenem antibiotics to decrease the level of serum VPA and we investigated in vitro transporting activity of VPA and VPA-Glu by P-gp. However, we could not detect any activity for VPA and VPA-Glu as substrates of P-gp, as shown in Table 3. The ATPase activity of Pgp at $33 \mu \mathrm{M}$ and $1.67 \mathrm{mM}$ VPA was $31.97 \pm 1.019$ and $30.59 \pm 2.110 \mathrm{nmol} / \mathrm{min} / \mathrm{mg}$ protein, respectively, and these values are the same value of minus substrates $33.78 \pm 0.237 \mathrm{nmol} / \mathrm{min} / \mathrm{mg}$ protein. VPA-Glu also did not show any ATPase activity as substrates for P-gp, of those values 31.26$34.23 \mathrm{nmol} / \mathrm{min} / \mathrm{mg}$, with the same value of minus substrates. Thus, we could not detect the substrate activity of VPA and VPA-Glu for P-gp. As a matter of course, MEPM at $12 \mu \mathrm{M}$ also showed no activation of P-gp. Thus, we could not study the influence

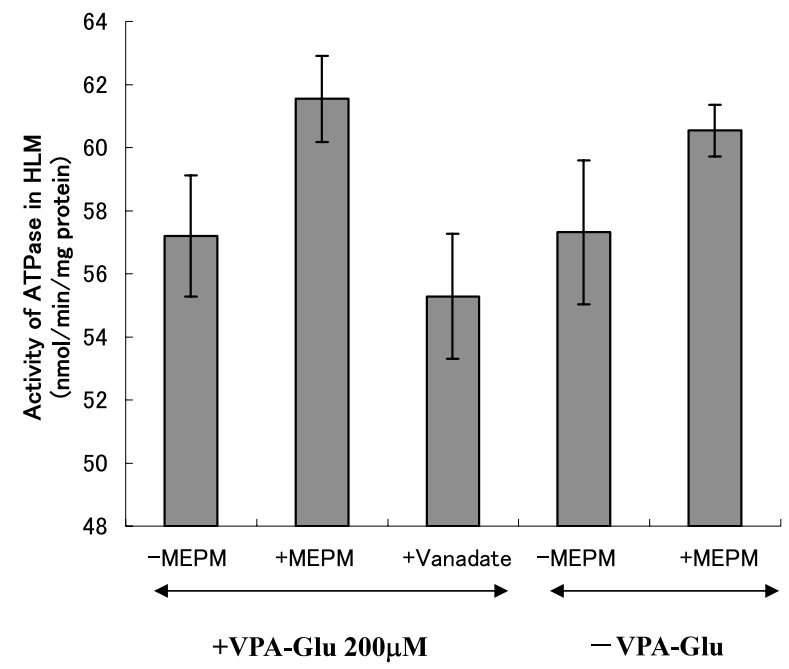

Fig. 6. Activation of ATPase in HLM with VPA-Glu in the Presence of MEPM

Those conditions to measure ATPase activity in HLM was described in the Method.

of MEPM on the activity of P-gp for transport of VPA and VPA-Glu.

We studied the transporting activity of VPAGlu by MRP2. MRP2 did not show any transporting activity of VPA-Glu as shown in Fig. 5. ATPase activity of VPA-Glu as substrates by MRP2 in the presence $(3.855 \pm 0.019 \mathrm{nmol} / \mathrm{min} / \mathrm{mg}$ protein) of $12 \mu \mathrm{M}$ MEPM is the same value with that of absence of MEPM $(3.930 \pm 0.063 \mathrm{nmol} / \mathrm{min} / \mathrm{mg}$ protein), as shown in lanes 1 (-MEPM) and 2 (+MEPM). This indicates that MEPM did not activate the MRP2 activity on membranes. The activity of MRP2 membranes is slightly more than the ATPase activity of control membranes. MRP2 membranes show ATPase activity by probenecid of a specific substrate for MRP2.

We measured (ATP-binding cassette ABC) transporting activity in HLM, which might contain all membranous activities. The results are shown in 
Table 4. No-activation of CYP2A6 and CYP3A4 by MEPM

\begin{tabular}{lccc}
\hline \hline \multirow{2}{*}{ CYP } & \multicolumn{3}{c}{ Activity $(\mathrm{nmol} / \mathrm{min} / \mathrm{mg}$ protein of HLM) } \\
\cline { 2 - 4 } & - MEPM & $20 \mu \mathrm{M}$ MEPM & $200 \mu \mathrm{M}$ MEPM \\
\hline CYP2A6 & $1.122 \pm 0.020$ & $1.095 \pm 0.057$ & $1.098 \pm 0.013$ \\
CYP3A4 & $0.385 \pm 0.023$ & $0.368 \pm 0.024$ & - \\
\hline
\end{tabular}

Fig. 6. We can detect slightly increase of ATPase activity in the presence of MEPM $(4.35 \mathrm{nmol} / \mathrm{min} / \mathrm{mg}$ protein). However, HLM contained high level of ATPase $(55-57 \mathrm{nmol} / \mathrm{min} / \mathrm{mg}$ protein) and we conclude no influence of MEPM on the transporters. We also studied with some ATPase inhibitors, such as EGTA and nitrilotriacetic acid, but it was difficult to see clear results. Recently, it has been reported that MRP4/5 plays a role of transport of VPA-Glu. ${ }^{14)}$ Thus, it is necessary to investigate with MRP4/5, although the experiment with the nephrectomized rats shows that kidney is not key organ for the decrease of serum VPA.

Study of the influence of MEPM and the activity of CYP2A6 and CYP3A4. We studied the effect of MEPM on CYP activities with HLM, but we could not find any difference between presence and absence of MEPM as shown in Table 4. We used the identical enzymes in the HLM for the measurement of activity of UGT and CYP. Cytochrome P450 in HLM was not activated by MEPM but UGT1A6 in HLM was activated by MEPM. We also confirmed no activation by MEPM with bovine liver microsomes. The specific activity of CYP2A6 was $0.292,0.287$ and $0.283 \mathrm{nmol} / \mathrm{min} / \mathrm{nmol}$ CYP in the absence, $30 \mu \mathrm{M}$ and $60 \mu \mathrm{M}$ MEPM, respectively. Thus, we concluded that MEPM did not influence on the CYP activity.

\section{DISCUSSION}

In order to understand the mechanisms of serum VPA decrease by carbapenem antibiotics, many research as shown in Table 1 was carried out in vivo with rats. Our biochemical experiment was carried out in vitro with human liver microsomes. This biochemical study will become strong skill for future study of the mechanisms of drug interaction. Our results might coincident with the phenomenon in a patient. The activation of VPA glucuronidation by MEPM was not found at $37^{\circ} \mathrm{C}$ but at $0^{\circ} \mathrm{C}$. We thought the protein conformation of UGT at $0^{\circ} \mathrm{C}$ with MEPM might be maintained in the stable state. It is possible that the in vitro activation by car- bapenem antibiotics may occur in patients. In our study, the used concentration of VPA and MEPM is the same or lower than the therapeutic level of those drugs. Thus, we believe that the activity increase of VPA glucuronidation by MEPM must occur in liver of patients.

Carbapenem antibiotics were used for the treatment of pneumonia when epileptic patients infected. MEPM has weak renal toxicity but IPM and PAPM have strong renal toxicity by hydrolyze with renal dehydroxypeptidase. Therefore, the enzyme inhibitiors such as cilastatin and betamipron were co-administered with IPM and PAPM, respectively. Thus, MEPM may be better in carbapenem antibiotics. The $\beta$-lactam antibiotics, such as imipenem and meropenem at $200-400 \mathrm{mg} / \mathrm{kg}$, have convulsive activity, as well as epilepsy diagnosis, and this depends upon the binding of carbapenem to $\gamma$-amino-butyric acid (GABA) receptor. ${ }^{21)}$ Perucca reported some interactions between anti-epileptic drugs, such as carbamazepine, phenytoin, phenobarbital, and primidone. ${ }^{22)}$ These drugs induce some drug-metabolizing enzymes and decrease the plasma level of these drugs. VPA inhibited phenobarbital and lamotrigine. Co-administration of antiepileptic drugs caused a neurotoxic effect. VPA induced hyperammonemia and thrombocytopenia in an elderly woman and caused the inhibition of carbamoyl-phosphate synthase. ${ }^{23)}$ From interactions between anti-epileptic and anti-psychotic drugs, chlorpromazine of an anti-psychotic drug possibly increases VPA concentration. ${ }^{24)}$

The serum valproic acid (dose of VPA, 400$1200 \mathrm{mg} /$ day) in epilepsy patients decreased by the administration of carbapenem antibiotics, such as meropenem, panipenem and imipenem. We surveyed the possible mechanisms explaining the decrease of the VPA level by carbapenem antibiotics as shown in Table 1. Studies to explain the mechanism were carried out by the following steps, such as absorption of VPA in the intestine, glucuronidation in the liver, disposition in blood and renal excretion. It is difficult to consider the inhibition of intestinal absorption, because carbapenem antibiotics are intravenously administered and do not reach the 
intestine at an effective concentration. The liver is the key organ for the decrease of VPA concentration by carbapenem antibiotics, because no decrease of the VPA level by carbapenem was found in hepatectomized rats but was found in nephrectomized rats. Yamamura et al. ${ }^{11)}$ showed that panipenem increased the level of hepatic UDPGA in rats. It is well known that UDPGA is made from UDPglucose by UDP-glucose dehydrogenase. UDPglucose is a house keeping substance like blood glucose and a main component in the root of glucose metabolism between glycogen and the TCA cycle. UDPGA is abundant and the concentration is some $\mathrm{mM}$; thus, it is difficult for us to agree with the changes of UDPGA concentration. Nakajima et al. ${ }^{12)}$ reported the mechanism of drug interaction between VPA and carbapenem antibiotics in monkeys and rats. UDPGA did not increase in monkey liver slices in the presence of carbapenem antibiotics. The most likely mechanism in liver is the activation of UGT by carbapenem antibiotics. We showed a 35\% increase of VPA-glucuronidation activity by the pre-incubation of human liver microsomes with meropenem. This increase fully compensates for the decrease of serum VPA level by carbapenem antibiotics. VPA-Glu is easily excreted from the kidney. Thus, we considered that the activation of VPA glucuronidation by carbapenem antibiotics is a key reaction.

VPA was not a ligand of P-gp. We considered that the decrease of VPA depends on the renal excretion of VPA-Glu and P-gp might play a role in this excretion. ${ }^{25)}$ We could not find for VPA-Glu to be a ligand of P-gp and MRP2 also did not show the ATPase activity, However, we found a little activity with HLM which contains high ATPase activity, therefore some other trasnporters, not P-gp or MRP2, may function to excrete VPA-Glu from kidney. Recently, it has been reported Mrp4/5 have a function to export VPA-Glu from erythrocytes. ${ }^{14)}$ Thus, it is possible that MRP4 may function to excrete VPA-Glu from kidney. However, transporters in kidney is not a key role to decrease serum VPA level, because nephrectomized rat has the function to decrease serum VPA and this suggests that kidney is not a target organ of the decrease VPA level by carbapenem antibiotics.

The pre-incubation of HLM with MEPM did not increase on the activities of CYP2A6 and CYP3A4, which present on microsomal membranes with UGT. UGT1A6 catalyzes glucuronidation of VPA and p-NP. MEPM activates glucuronida- tion of VPA and p-NP depending on UGT1A6. MEPM also activates glucuronidation of androsterone by UGT2B7. Thus, MEPM did not activate cytochrome P450 but UGT. Meanwhile, this work did not clarify the specific activation of VPA by carbapenem antibiotics, because the activation by MEPM was found not only on UGT1A6 (VPA and p-NP) but also on UGT2B7 (androsterone) as shown in Table 2. Perucca ${ }^{22)}$ and Besag et al. ${ }^{24)}$ described many interactions between antiepileptic and antipsychotic drugs. We found no activation of UGT1A6 by streptopmycin but found weak activity increase by ampicillin. Meanwhile, in clinical case reports, ${ }^{7,25)}$ ampicillin and cephalosporin amtoniotics did not influence on plasma VPA level. These three antibiotics (carbapenem, penicillin, and cephalosporin) have similar structure as shown in Fig. 1, but carbapenem antibiotics among three antibiotics have the decreasing activity of serum VPA level. The mechanism of activation of UGT by MEPM is not clarified but it is possible that MEPM at therapeutic concentration activates directly UGT molecules by chaperon-like activity or indirectly by stabilization change in membrane structure. The mechanism of the activity increase of glucuronidation will be studied in our future plan.

Acknowledgements This review was initiated by the comments by Prof. Hiroaki Yuasa. Human MRP2 was generously supplied from GenoMembrane, Tsurumi-ku, Yokohama, Japan.

\section{REFERENCES}

1) Mizutani, T. (2003) PM frequencies of major CYPs in asians and caucasians. Drug Metab. Rev., 35, 99106.

2) Radomynska-Pandya, A., Czernik, P. J., Little, J. M., Battaglia, E. and Mackenzie, P. I. (1999) Structural and functional studies of UDPglucuronosyltransferases. Drug Metab. Rev., 31, 817-899.

3) Usui, T., Kuno, T., Ueyama, H., Ohkubo, I. and Mizutani, T. (2006) Proximal HNF1 element is essential for the induction of human UDPglucoronosyltransferase $1 \mathrm{~A} 1$ by glucocorticoid receptor. Biochem. Pharmacol., 71, 693-701.

4) Negishi, M., Pedersen, L. G., Petrotchenko, E., Shevtsov, S., Gotokhov, A., Kakuta, Y. and Pedersen, L. C. (2001) Structure and function of sulfotransferases. Arch. Biochem. Biophys., 390, 149- 
157.

5) Mizutani, T. and Hattori, A. (2005) New horizon of MDR1 (P-glycoprotein) study. Drug Metab. Rev., 37, 489-510.

6) Remmer, H., Schoene, B. and Fleischmann, R. A. (1973) Induction of the unspecific microsomal hydroxylase in the human liver. Drug Metab. Dispos., 1, 224-230.

7) Nagai, K., Shimizu, T., Togo, A., Takeya, M., Yokomizo, Y., Sakata, Y., Matsuishi, T. and Kato, H. (1997) Decrease in serum levels of valproic acid during treatment with a new carbapenem, panipenem/betamipron. J. Antimicrob. Chemother., 39, 295-296.

8) Kojima, S., Nadai, M., Kitaichi, K., Wang, L. I., Nabeshima, T. and Hasegawa, T. (1998) Possible mechanism by which the carbapenem antibiotic panipenem decreases the concentration of valproic acid in plasma in rats. Antimicrob. Agents Chemother., 42, 3136-3140.

9) Torii, M., Takiguchi, Y., Saito, F., Izumi, M. and Yokota, M. (2001) Inhibition by carbapenem antibiotic imipenem of intestinal absorption of valproic acid in rats. J. Pharm. Pharmacol., 53, 823-829.

10) Yamamura, N., Imura, K., Naganuma, H. and Nishimura, K. (1999) Panipenem, a carbapenem antibiotic, enhances the glucuronidation of intravenously administered valproic acid in rats. Drug Metab. Dispos., 27, 724-730.

11) Yamamura, N., Imura-Miyoshi, K. and Naganuma, H. (2000) Panipenem, a carbapenem antibiotic, increases the level of hepatic UDP-glucuronic acid in rats. Drug. Metab. Dispos., 28, 1484-1486.

12) Nakajima, Y., Mizobuchi, M., Nakamura, M., Takagi, H., Inagaki, H., Kominami, G., Koike, M. and Yamaguchi, T. (2004) Mechanism of the drug interaction between valproic acid and carbapenem antibiotics in monkeys and rats. Drug Metab. Dispos., 32, 1383-1391.

13) Omoda, K., Murakami, T., Yumoto, R., Nagai, J., Maeda, Y., Kiribayashi, Y. and Takano, M. (2005) Increased erythrocyte distribution of valproic acid in pharmacokinetic interaction with carbapenem antibiotics in rat and human. J. Pharm. Sci., 94, 16851693.

14) Ogawa, K., Yumoto, R., Hamada, N., Nagai, J. and Takano, M. (2006) Interaction of valproic acid and carbapenem antibiotics with multidrug resistanceassociated proteins in rat erythrocyte membranes.
Epilepsy Res., 71, 76-87.

15) Yokogawa, K., Iwashita, S., Kubota, A., Sasaki, Y., Ishizaki, J., Kawahara, M., Matsushita, R., Kimura, K., Ichimura, F. and Miyamoto, K. (2001) Effect of meropenem on disposition kinetics of valproate and its metabolites in rabbits. Pharm. Res., 18, 13201326.

16) Kanou, M., Saeki, K., Kato, T., Takahashi, K. and Mizutani, T. (2002) Study of in vitro glucuronidation of hydroxyquinolines with bovine liver microsomes. Fundam. Clin. Pharmacol., 16, 513-517.

17) Sarkadi, B., Price, E. M., Boucher, R. C., Germann, U. A. and Scarborough, D. A. (1992) Expression of the human multidrug resistance cDNA in insect cells generates a high activity drug-stimulated membrane ATPase. J. Biol. Chem., 267, 4854-4858.

18) Walseth, T. F. and Johnson, R. A. (1979) The enzymatic preparation of $\left[\gamma_{-}{ }^{32} \mathrm{P}\right]$ nucleotide triphosphate, cyclic $\left[{ }^{32} \mathrm{P}\right] \mathrm{AMP}$, and cyclic GMP. Biochim. Biophys. Acta, 526, 11-31.

19) Hirano, Y., Uehara, M., Saeki, K., Kato, T., Takahashi, K. and Mizutani, T. (2002) The influence of quinolines on coumarin 7-hydroxylation in bovine liver misrosomes and human CYP2A6. $J$. Health Sci., 48, 118-125.

20) Hirano, Y. and Mizutani, T. (2003) Study of inhibition of CYP2A6 by some drugs derived from quinoline. J. Pharm. Pharmacol., 55, 1667-1672.

21) Horiuchi, M., Kimura, M., Yokumura, M., Hasebe, N., Arai, T. and Abe, K. (2006) Absence of convulsive liability of doripenem, a new carbapenem antibiotic, in comparison with $\beta$-lactam antibiotics. Toxicology, 222, 114-124.

22) Perucca, E. (2006) Clinically relevant drug interactions with antiepileptic drugs. Br. J. Clin. Pharmacol., 61, 246-255.

23) Mallet, L., Babin, S. and Morais, J. A. (2004) Valproic acid-induced hyperammonia and thrombocytopenia in an elderly woman. Ann. Pharmacother., 38, 1643-1647.

24) Besag, F. M. and Berry, D. (2006) Interactions between antiepileptic and antipsychotic drugs. Drug Saf., 29, 95-118.

25) De Turck, B. J. G., Diltoer, M. W., Cornelis, P. J. W. W., Maes, V., Spapen, H. D. M., Camu, F. and Huyghens, L. P. (1998) Lowering of plasma valproic acid concentrations during concomitant therapy with meropenem and amikacin. J. Antimicrob. Chemother., 42, 563-564. 